\title{
Sagittal adjusting screws for the correction of grade IV spondylolisthesis in a patient with Ehlers-Danlos syndrome: illustrative case
}

\author{
Jake Jasinski, DO, Doris Tong, MD, MSc, FRCPC, Connor Hanson, PSM, and Teck Soo, MD, FRCSC \\ Division of Neurosurgery, Ascension Providence Hospital, College of Human Medicine, Michigan State University, Southfield, Michigan
}

BACKGROUND Ehlers-Danlos syndrome (EDS) and its connective tissue laxity often result in high-grade lumbosacral spondylolisthesis. Patients present with debilitating symptoms and neurological deficits. Reports of surgical techniques in non-EDS patients for the treatment of high-grade lumbosacral spondylolisthesis mainly described an open approach, multilevel fusions, and multiple stages with different circumferential approaches. Sagittal adjusting screws (SASs) can be used in a minimally invasive (MI) fashion, allowing intraoperative reduction.

OBSERVATIONS A 17-year-old female with EDS presented to the authors' institute with severe lower back and left L5 radicular pain in 2017 . She presented with a left foot drop and difficulty ambulating. Magnetic resonance imaging showed grade IV L5-S1 spondylolisthesis. She underwent lumbar fusion for intractable back pain with radiculopathy. Intraoperatively, percutaneous SASs and extension towers were used to distract the L5-S1 disc space and reduce the spondylolisthesis. MI transforaminal lumbar interbody fusion was completed with significant symptomatic relief postoperatively. The patient was discharged to home 3 days postoperatively. Routine follow-up visits up to 3 years later demonstrated solid fusion radiographically and favorable patient-reported outcomes.

LESSONS The authors used SASs in a MI approach to successfully correct and stabilize grade IV spondylolisthesis in an EDS patient with a favorable long-term patient-reported outcome.

https://thejns.org/doi/abs/10.3171/CASE21196

KEYWORDS spine surgery; Ehlers-Danlos syndrome; grade IV spondylolisthesis; sagittal adjusting screw; minimally invasive

Ehlers-Danlos syndrome (EDS) is an inherited connective tissue disorder due to collagen malformation. Patients diagnosed with EDS experience joint laxity that often leads to bony dislocations and musculoskeletal injuries. ${ }^{1}$ As a result, patients diagnosed with EDS often develop spinal scoliotic deformities and high-grade spondylolisthesis. While the surgical management of appendicular skeletal manifestations of patients with EDS is well described, there is a lack of literature that describes the surgical management of high-grade spondylolisthesis in patients with EDS. A PubMed search performed on March 18, 2021, regarding surgical treatment of spondylolisthesis in EDS revealed 2 case reports. Our search syntax is listed in the Appendix.

High-grade lumbosacral spondylolisthesis can be a challenging surgical pathology. Patients often present with debilitating symptoms along with neurological deficits. ${ }^{2,3}$ With the joint laxity associated with EDS, there can be a dynamic exacerbation of the spondylolisthesis grade. ${ }^{4}$ To date, surgical techniques to treat high-grade spondylolisthesis in EDS patients have rarely been described. ${ }^{4}$ Previous studies involving patients without EDS described different surgical procedures. The majority were open approach multilevel fusions using multiple stages with different circumferential approaches. ${ }^{5,6}$ Besides, these techniques only achieved stabilization fusion without spondylolisthesis correction. In some of these fusions, instrumentation was not used. ${ }^{7,8}$

The minimally invasive (MI) sagittal adjusting screw (SAS) system was first introduced in 2013 by Medtronic to correct spinal deformity secondary to fractures. ${ }^{9}$ We believe the correction mechanisms of the

ABBREVIATIONS CT = computed tomography; EDS = Ehlers-Danlos syndrome; EMG = electromyography; $\mathrm{MI}=$ minimally invasive; $\mathrm{MRI}=$ magnetic resonance imaging; ODI = Oswestry Disability Index; PCS = physical component score; PEEK = polyetheretherketone; SAS = sagittal adjusting screw; SF-12 = 12-item Short Form Health Survey; TLIF = transforaminal lumbar interbody fusion.

INCLUDE WHEN CITING Published July 12, 2021; DOI: 10.3171/CASE21196.

SUBMITTED April 1, 2021. ACCEPTED May 11, 2021.

(C) 2021 The authors, CC BY-NC-ND 4.0 (http://creativecommons.org/licenses/by-nc-nd/4.0/). 
SAS system will be similarly helpful in this patient. In this study, we sought to demonstrate that it is possible to reduce and stabilize grade IV spondylolisthesis in a patient with EDS through a 1-stage, singlelevel, MI transforaminal lumbar interbody fusion (TLIF) using the SAS system with a favorable long-term outcome. This case report was written following the CARE case report guidelines.

\section{Illustrative Case}

\section{Patient Information}

The patient was a 17-year-old female with a history of EDS. She was a highly active teenager and an accomplished gymnast. She had first experienced low back pain and bilateral $L 5$ radicular pain in 2015. Her symptoms had significantly limited her physical activity. No other history was contributory.

\section{Clinical Findings}

The patient first presented in 2017 with bilateral lower extremity radicular pain after 1 year of failed physical therapy. Her preoperative 12-item Short Form Health Survey (SF-12) physical component score (PCS) was 32.1, and her Oswestry Disability Index (ODI) was 54. On examination, the patient had a left foot drop. Her ambulation was significantly limited. She did not have any other neurological deficits.

\section{Diagnostic Assessment}

The patient's lumbar computed tomography (CT) and magnetic resonance imaging (MRI) revealed a grade IV L5 on S1 anterolisthesis secondary to a bilateral pars defects, causing severe central and bilateral foraminal stenosis (Fig. 1). The prognosis for high-grade spondylolisthesis is widely debated and ranges from full asymptomatic recovery to progressive neurological deficits with appropriate therapy. ${ }^{10}$ We offered her L5-S1 decompression and instrumentation fusion with an SAS system to address her spinal instability and deformity.

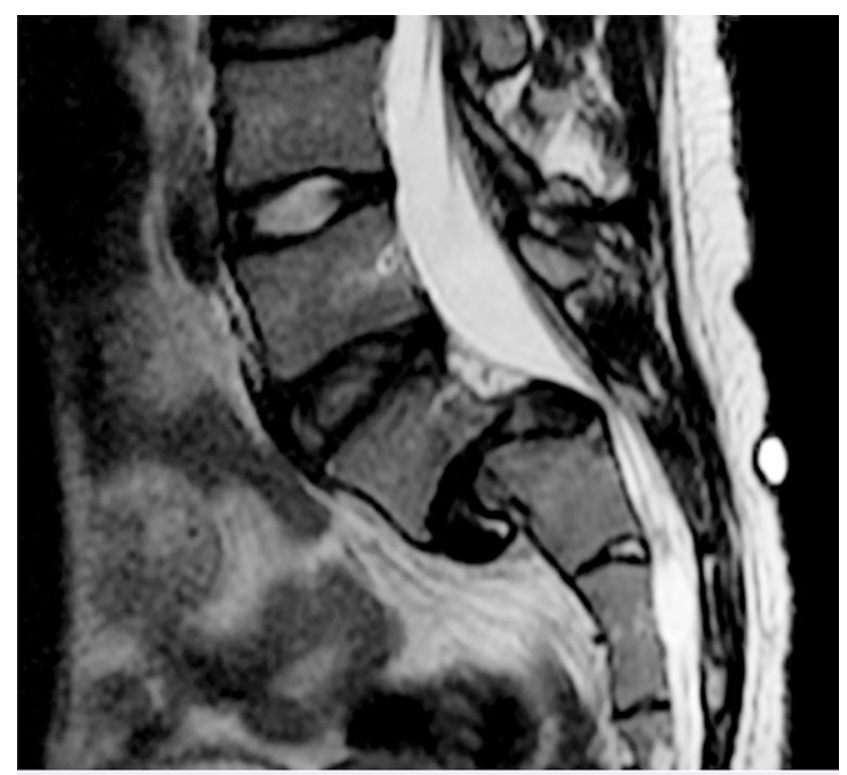

FIG. 1. Preoperative MRI of the lumbar spine without contrast. Grade IV spondylolisthesis is noted at L5-S1, leading to severe central stenosis.

\section{Therapeutic Intervention}

The SAS is a monoaxial screw with a fixed head. Unlike typical monoaxial screws, the SAS head contains a sliding saddle that is concave and accommodates the lordosis of the rod in the sagittal plane once inserted. Together with the compressor/distractor and fracture reducer devices that attach to the SASs' percutaneous extension towers, distraction force and lordosis can be applied at the instrumented levels and at both the kyphotic and lordotic curves of the spine to correct spondylolisthesis.

\section{Surgical Technique}

The patient was placed in a prone position on a Jackson table. Before positioning, electromyography (EMG) was performed to monitor the L5 nerve root. Intraoperative fluoroscopy was used to assess the patient's spinal deformity after her positioning, and we noted her grade IV spondylolisthesis had progressed to a grade V deformity.

We placed 4 Kirschner wires into the pedicles of $L 5$ and S1 using anteroposteriorly and laterally positioned $\mathrm{C}$-arms and then placed percutaneous SASs and their corresponding screw extender towers into the right-sided pedicles of L5 and S1. We achieved a bicortical purchase. The distractor/ compressor device was then attached to connect at the base of the L5 and S1 screw extender towers. The fracture reducer device was subsequently placed at the top of the screw extender devices. The final SAS system with distractor/compressor and fracture reducer device assembly is shown in Fig. 2. The distractor/compressor device applied a perpendicular force at the base of the screw extenders to distract the L5-S1 disc space under continuous fluoroscopy. Once adequate distraction was achieved, we used the fracture reducer device to induce lordosis at the instrumented levels (Fig. 3A). This part of the procedure was carried out under continuous fluoroscopy. We then percutaneously placed a temporary hyperlordotic rod through the tulip heads of the right-sided SAS. The temporary rod's length was oversized to increase the force used to reduce the patient's
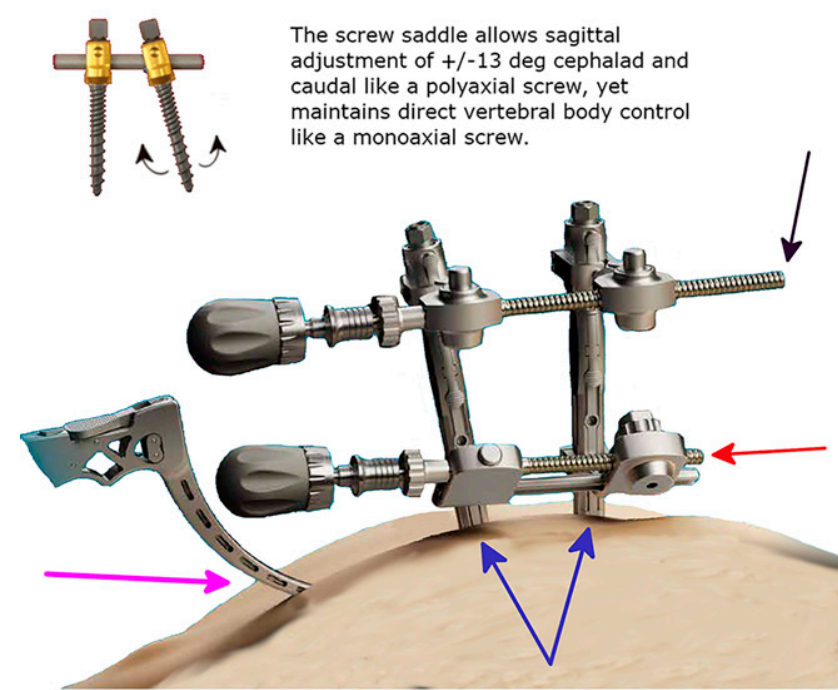

FIG. 2. SAS system with distractor/compressor and fracture reducer devices. Fracture reducer (black arrow) induces lordosis while the distractor/compressor (red arrow) distracts the disc space at the instrumented L5 and S1 vertebrae. These devices attach to the screw extension towers (blue arrows) attached to the right-sided pedicle screws at L5 and S1. The rod is inserted percutaneously using the rod holder (pink arrow). The inset picture shows the saddle mechanism of the SAS screw and explanatory text. 

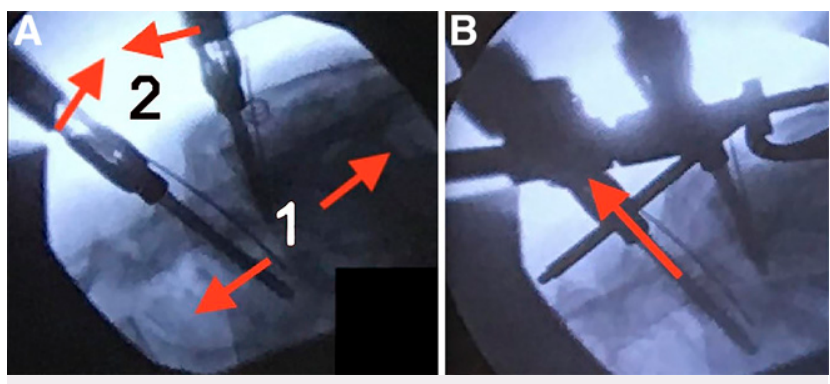

FIG. 3. A: Intraoperative fluoroscopy with right-sided SASs before applying distraction and lordosis. First, distraction is applied at the disc space (1; red arrows indicate distraction force applied at the L5 and $\mathrm{S} 1$ vertebral bodies), and then a force perpendicular to the screw extender towers is applied to induce lordosis (2; red arrows indicate force applied perpendicular to the screw extenders that induces lordosis at L-S1). B: Intraoperative fluoroscopy after distracting and applying lordosis while using the rod to reduce the spondylolisthesis. Red arrow indicates the force vector introduced by the surgeon on the $L 5$ pedicle screw and rod to reduce the spondylolisthesis of $L 5$ on $S 1$.

spondylolisthesis. The sliding saddle at the head of the SASs accommodated the temporary rod's extreme lordosis. We then used a locking nut to secure the base of the rod at the S1 tulip head. We left the distal end of the rod proud above the right $L 5$ tulip head to reduce the spondylolisthesis at L5-S1. While keeping the rod proud at L5, we slowly introduced a locking nut to secure the temporary rod to the $L 5$ tulip head. The tightening of the locking cap onto the rod slowly pulled the $L 5$ vertebra back into alignment with S1. We could pull the L5 vertebra back onto the S1 vertebra and reduce the patient's condition to grade I spondylolisthesis (Fig. 3B). We performed the preceding steps under continuous fluoroscopy with EMG to avoid injuring the exiting $L 5$ nerve root. We could have further reduced the spondylolisthesis; however, we began to observe EMG changes consistent with $\mathrm{L} 5$ nerve root compression, which caused us to stop further reduction.

We tightened the $\mathrm{L} 5$ locking nut until we secured the temporary rod into the right L5 and S1 SAS tulip heads. The temporary rod was used to maintain disc space distraction, lordotic angle, and reduction at L5-S1 to perform the interbody fusion.

A MI TLIF using a tubular retraction system was then performed from the left side. A polyetheretherketone (PEEK) interbody cage was used. Recombinant human bone morphogenetic protein 2 was placed inside the cage along with autograft. Cancellous allograft was inserted into the interbody space surrounding the PEEK interbody cage. After decompression and cage implantation, we inserted multiaxial screws at the left L5 and S1 vertebrae. We then percutaneously placed a lordotic rod and fastened it to the left-sided L5 and S1 screws, completing our left-sided construct. Subsequently, we removed the right-sided temporary rod. An appropriately sized lordotic rod was inserted and secured with locking caps to the rightsided L5 and S1 SASs. We concluded the surgery after 146 minutes with $200 \mathrm{~mL}$ of estimated blood loss.

\section{Follow-Up and Outcomes}

The patient's radicular pain improved immediately postoperatively. She was ambulating on postoperative day 1. Her pain and ambulation improved throughout her 3 days of hospital stay. She did not require any further physical therapy upon discharge.

We conducted clinical and radiographic follow-up of the patient (Fig. 4). At 6 months and 1 year, her foot drop had resolved; her SF-12 PCSs at those visits were 30.6 and 46.9, respectively; and her ODI values were 2 and 0 , respectively. One year postoperatively, the patient began to complain of palpable hardware and requested hardware removal. Two-year postoperative lumbar MRI and 3-year postoperative lumbar CT (Fig. 5) demonstrated grade I spondylolisthesis with solid bony interbody fusion.

\section{Discussion}

\section{Observations}

EDS is a rare hereditary condition affecting connective tissues leading to hypermobility of the joints, hyperextensibility of the skin, poor healing, and bruising. The spinal conditions include ligamentous laxity, dislocated joints, scoliosis, and high-grade spondylolisthesis. ${ }^{11}$

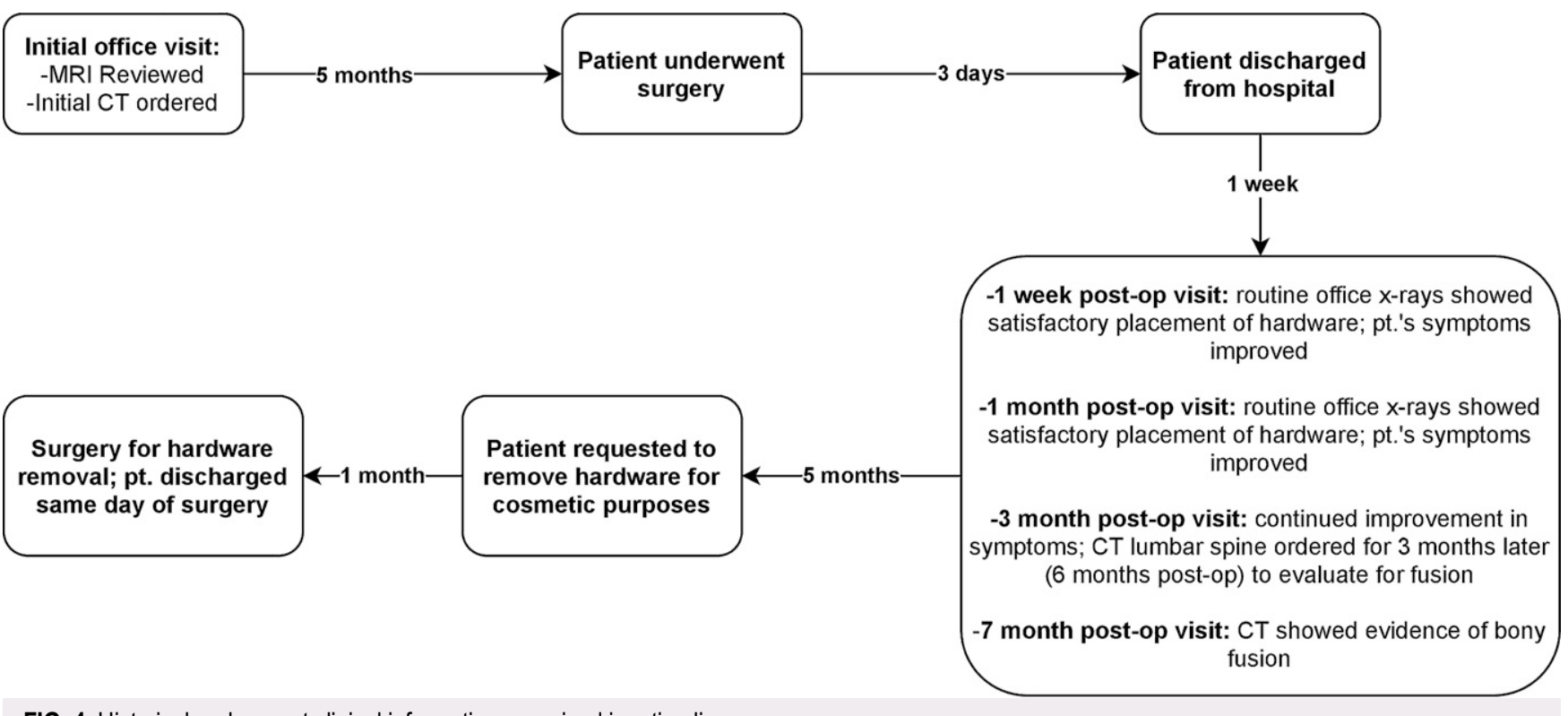

FIG. 4. Historical and current clinical information organized in a timeline. 


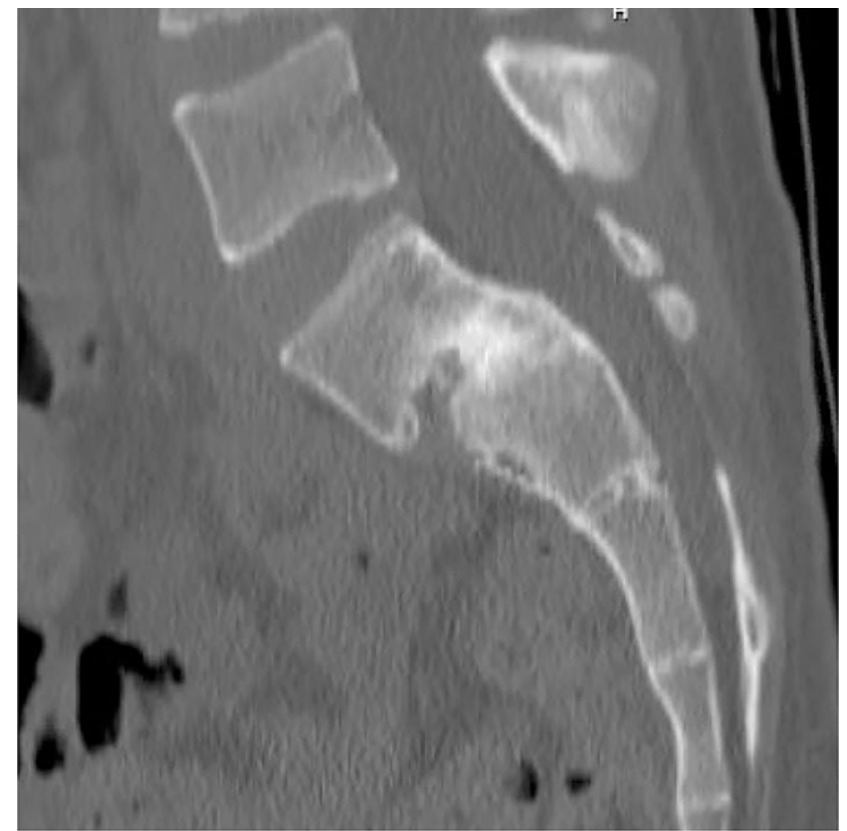

FIG. 5. Sagittal image from a CT scan of the lumbar spine without contrast at 3 years after surgery. Reduction to grade I spondylolisthesis is noted with improvement in central stenosis. Osseous bridging is again noted at the interbody space.

High-grade lumbosacral spondylolisthesis is associated with debilitating symptoms along with neurological deficits. ${ }^{2,3}$ Surgical treatment of this condition in EDS patients has been rarely reported.

In non-EDS patients, addressing high-grade spondylolisthesis has often involved a 2-stage anterior and posterior approach, and an open posterior approach has also been reported. ${ }^{12-14}$ Open surgical techniques were associated with significant morbidities. ${ }^{15-17}$ Compared with open spinal fusion surgery, Ml spinal surgery is associated with less tissue injury, less blood loss, and shorter hospital stay, whereas the benefits regarding complications and functional outcomes remain inconclusive. ${ }^{15-17}$ Shedid et al. described a MI procedure using TLIF at L4-5 and a transsacral rod to fuse and stabilize the high-grade lumbosacral spondylolisthesis without achieving spondylolisthesis correction. ${ }^{8}$ The SAS system, a fifthgeneration MI spinal fusion system introduced in 2013, includes fixed-head saddle pedicle screws, a compressor/distractor, and fracture reducer devices, which allows surgeons to position the SAS in the natural kyphotic and lordotic curves of the spine in the correction of complex spinal pathologies. In this case report, we demonstrated the SAS system's use to distract, apply lordosis, and reduce spondylolisthesis grade in an EDS patient with high-grade lumbosacral spondylolisthesis through a 1-stage, MI, single-level TLIF with a favorable long-term patient-reported outcome.

Although the patient's joint laxity and hypermobility associated with her EDS may have been the cause of her high-grade spondylolisthesis, her spondylolisthesis reduction was aided by the joint laxity at the intervertebral disc and bilateral facet joints, a condition not generalizable to the general population. We were also able to use SASs unilaterally instead of bilaterally to distract, apply lordosis, and reduce the L5-S1 segment due to the patient's normal bone density and lack of degenerative changes. When generalized to a different patient population, such as patients with osteoporosis or increased body mass index, the significant force vector applied to unilateral SASs to correct the spondylolisthesis risks pulling out of the SASs in patients with weaker bone structure, and correction with bilateral SASs may be necessary. With bilateral SASs, the force vectors were distributed over an increased number of screws, preventing pullout risk.

\section{Lessons}

With this case report, we have demonstrated use of the SAS system in an EDS patient with a posterior approach and a 1-stage, single-level, MI surgical technique as an alternative to the more invasive and extensive surgical techniques to reduce high-grade lumbosacral spondylolisthesis. We achieved solid fusion with a favorable long-term patient-reported outcome. However, the use of this technique in non-EDS patients must be further investigated.

\section{Appendix}

The following search syntax were used for our PubMed search conducted on 2021-3-18:

((spinal instability) AND ((Ehlers-Danlos Syndrome OR Ehlers Danlos Syndrome)))

(spondylolisthesis) AND (Ehlers Danlos Syndrome OR EhlersDanlos Syndrome OR EDS)

(spondylolisthesis) AND (Ehlers Danlos Syndrome OR EhlersDanlos Syndrome)

(spondylolisthesis) AND (Ehlers Danlos Syndrome)

\section{Acknowledgments}

We thank Dr. Boyd Richards and Brian McCormack for their contribution to this case study.

\section{References}

1. Fang H, Liu P-F, Ge C, et al. Anterior cervical corpectomy decompression and fusion for cervical kyphosis in a girl with Ehlers-Danlos syndrome: a case report. World J Clin Cases. 2019;7(4):532-537.

2. Fabris DA, Costantini $S$, Nena U. Surgical treatment of severe L5-S1 spondylolisthesis in children and adolescents. Results of intraoperative reduction, posterior interbody fusion, and segmental pedicle fixation. Spine (Phila Pa 1976). 1996;21(6):728-733.

3. Harris IE, Weinstein SL. Long-term follow-up of patients with gradeIII and IV spondylolisthesis. Treatment with and without posterior fusion. J Bone Joint Surg Am. 1987;69(7):960-969.

4. Acosta FL Jr, Ames CP, Chou D. Operative management of adult high-grade lumbosacral spondylolisthesis. Neurosurg Clin N Am. 2007;18(2):249-254.

5. DeWald CJ, Vartabedian JE, Rodts MF, Hammerberg KW. Evaluation and management of high-grade spondylolisthesis in adults. Spine (Phila Pa 1976). 2005;30(6 suppl):S49-S59.

6. Beck AW, Simpson AK. High-grade lumbar spondylolisthesis. Neurosurg Clin N Am. 2019;30(3):291-298.

7. Nematbakhsh $\mathrm{A}$, Crawford $\mathrm{AH}$. Non-adjacent spondylolisthesis in Ehlers-Danlos syndrome. J Pediatr Orthop B. 2004;13(5):336-339.

8. Shedid D, Weil AG, Lieberman I. A novel minimally invasive technique for the treatment of high-grade isthmic spondylolisthesis using a posterior transsacral rod. J Spinal Disord Tech. 2014;27(2): E41-E48.

9. Rocha V, Warren J. Medtronic Announces new screw for marketleading CD HORIZON solera spinal system. Medtronic. July 11, 2013. Accessed March 29, 2021. https://news.medtronic.com/ 2013-07-11-Medtronic-Announces-New-Screw-for-Market-LeadingCD-HORIZON-R-SOLERA-R-Spinal-System 
10. Emary PC, Eberspaecher SA, Taylor JA. High-grade spondylolytic spondylolisthesis. J Can Chiropr Assoc. 2017;61(2):162-166.

11. Miklovic T, Sieg VC. Ehlers Danlos syndrome. StatPearls. 2021. Accessed March 29, 2021. http://www.ncbi.nlm.nih.gov/books/ NBK549814/

12. Elnokaly MA, Adawi MM, Nabeel AM. Pedicular lumbosacral spine fusion for adult/adolescent lumbar developmental high-grade spondylolisthesis. Surg Neurol Int. 2020;11:416.

13. Kalani MA, Kouloumberis $P$, Richards $A E$, et al. Retrospective radiographic analysis of anterior lumbar fusion for high grade lumbar spondylolisthesis. J Spine Surg. 2020;6(4):650-658.

14. Moreau $S$, Lonjon $G$, Guigui $P$, et al. Reduction and fusion in highgrade L5-S1 spondylolisthesis by a single posterior approach. Results in 50 patients. Orthop Traumatol Surg Res. 2016;102(2):233-237.

15. Park Y, Ha JW, Lee YT, Sung NY. Minimally invasive transforaminal lumbar interbody fusion for spondylolisthesis and degenerative spondylosis: 5-year results. Clin Orthop Relat Res. 2014;472(6):1813-1823.

16. Khan NR, Clark AJ, Lee SL, et al. Surgical outcomes for minimally invasive vs open transforaminal lumbar interbody fusion: an updated systematic review and meta-analysis. Neurosurgery. 2015;77(6):847-874.

17. Hammad A, Wirries $A$, Ardeshiri $A$, et al. Open versus minimally invasive TLIF: literature review and meta-analysis. J Orthop Surg Res. 2019;14(1):229.

\section{Disclosures}

The authors report no conflict of interest concerning the materials or methods used in this study or the findings specified in this paper.

\section{Author Contributions}

Conception and design: Jasinski, Soo. Acquisition of data: Jasinski. Analysis and interpretation of data: Jasinski. Drafting the article: Jasinski, Hanson. Critically revising the article: all authors. Reviewed submitted version of manuscript: all authors. Approved the final version of the manuscript on behalf of all authors: Jasinski. Administrative/technical/ material support: Jasinski. Study supervision: Jasinski, Tong, Soo.

\section{Correspondence}

Jake Jasinski: Ascension Providence Hospital, College of Human Medicine, Michigan State University, Southfield, MI. jake.m.jasinski@ gmail.com. 